\title{
Medidor de actividad eléctrica muscular cuatro canales electromiógrafo (EMG) inalámbrico
}

\section{Wireless four-channel electromyograph (EMG) for the measurement of electircal muscle activity}

José de Jesús Salgado P. ${ }^{[1]}$, Lismar Tobías Córdoba M. ${ }^{[2]}$ y Michael Javier Dussan M. ${ }^{[3]}$

\begin{abstract}
Resumen
Este articulo presenta el desarrollo de un prototipo de registro electromiográfico de cuatro canales, portátil, inalámbrico y de bajo consumo, el cual está conformado por múltiples etapas de desarrollo, con la capacidad de registrar, visualizar y procesar señales electromiográficas superficiales de los músculos en extremidades superiores e inferiores del cuerpo humano. El tipo de comunicación que se implementó para la transmisión de datos desde el prototipo hacia el computador es la comunicación serial RS232 inalámbrica, por tal motivo se utiliza el módulo XBEE en modo transparente, finalmente a las señales electromiógraficas se aplica la Transformada Wavelet Continua por medio del programa de LabVIEW, para obtener el espectro de las frecuencias principales, característica necesarias para detectar patrones de movimientos de los músculos y detectar así posibles deficiencias o rendimiento muscular.
\end{abstract}

Palabras clave: EMGs, RS232, LABVIEW, XBEE, CWT, wavelet, inalámbrico.

\begin{abstract}
This article presents the multiple stages of the development of a low-power pocket four-channel electromyography prototype, able to record, display and process surface electromyographic signals that originate in the muscles in the top and bottom limbs. Wireless RS232 serial communication and XBee module in transparent mode were implemented for the transmission of data from the prototype to the computer. Finally, Continuous Wavelet Transform was applied to the electromyography signals via LabVIEW to obtain the main frequency spectrum, needed to detect patterns and muscle movements and identify any muscle weaknesses or inadequate performance.
\end{abstract}

Key words: EMGs, RS232, LABVIEW, XBEE, CWT, wavelet, wireless

[1] Ingeniero Electrónico. Universidad Surcolombiana. Neiva Av. Pastrana Borrero - Carrera 1. josesalgadop@usco.edu.co [2] Ingeniero Electrónico. Universidad Surcolombiana. Neiva Av. Pastrana Borrero - Carrera 1. Ramsil56@hotmail.com

[3] Ingeniero Electrónico. Universidad Surcolombiana. Neiva Av. Pastrana Borrero - Carrera 1. maicoljdmunoz@hotmail.es

Recibido: 29 enero 2015 • Aceptado: 30 abril 2015. 


\section{Introducción}

Actualmente las señales mioeléctricas son utilizadas principalmente en el diagnóstico médico para detectar patologías humanas. Sin embargo, cada día en el campo de las tecnologías médicas se da un gran avance con el desarrollo de equipos más sofisticados, pero su costo dificulta a los profesionales de la medicina el uso de estos avances (Soto, 2009). Las señales mioeléctricas también pueden ser utilizadas en muchas áreas de aplicación como en la industria, en el hogar, en el entretenimiento, en el control de interfaces hombremáquina, tales como prótesis mioeléctricas, robot móviles, brazos robóticos o brazos simulados mediante software, constituyendo aplicaciones en las que tienen cabida el control utilizando señales mioeléctricas (Muñoz, 2004).

En Colombia se ha observado el uso de las señales mioeléctricas aplicadas en diversos campos de la industria y la medicina, tales como el desarrollo de aplicaciones en control de prótesis de mano (Realpe, 2007) y de miembros inferiores por el aumento repentino de las cifras de amputados en Colombia resultado del conflicto interno. Además, se han usado las señales mioeléctricas en el registro de la actividad eléctrica de los músculos y en la estimulación eléctrica funcional para la rehabilitación de pacientes con parálisis por influencia de la fatiga muscular (Fernández, 2009), en aplicaciones de las señales mioeléctricas para el control de interfaces hombre-máquina (Muñoz, 2004), en exoesqueletos para potenciar las capacidades humanas y apoyar la rehabilitación (Chávez, 2010). Pero este tipo de investigación se ve afectado por el poco apoyo por parte del gobierno en los últimos cincos años, ya que la inversión en actividades tales como en la ciencia, tecnología e innovación a nivel nacional es del 0.44\% del PIB y del 0.2\% del PIB en inversión y desarrollo de tecnologías (Salazar, 2011).

El propósito de este proyecto es realizar un trabajo de aplicación, relacionado con el desarrollo de un sistema de registro de las señales mioeléctricas superficiales (EMGS) del cuerpo humano de cuatro canales, con la capacidad de transmitir la señal procesada por medio de un dispositivo de comunicación inalámbrica, que se encargue de hacer un enlace remoto entre el dispositivo EMG y una computadora, que cuente con un sistema de procesamiento digital para la visualización de la señales y el respectivo análisis frecuencial de estas. El sistema se debe caracterizar por su bajo consumo de potencia, bajo costo y permitir obtener resultados satisfactorios en el análisis de la actividad eléctrica de los músculos, además de presentar una alta sensibilidad, precisión, exactitud.

\section{Metodología}

Este proyecto se dividió en fases para su proceso y desarrollo. Las fases desarrolladas fueron:

\subsection{Etapa de adquisición y amplificación de la señal}

Debido a los niveles bajos de voltajes generados en los músculos las señales se deben acondicionar a un nivel óptimo, se requiere de un sistema que pueda ser capaz de amplificar este tipo de voltajes. Para tal propósito se diseñó un sistema de adquisición y amplificación analógica de las señales mioeléctricas.

El sistema detecta y registra potenciales de acción de los músculos sobre la superficie de la piel, por lo cual se implementan electrodos superficiales de cloruro de plata 3M (Figura 1), con un rango de voltaje referencial de $100 \mu \mathrm{V}$ a $100 \mathrm{mV}$. Se tiene en cuenta el uso de este tipo de electrodos por ser no invasivos, desechables, cómodos y de muy bajo costo, también tienen una baja impedancia en comparación a otros electrodos del mercado. Para obtener lecturas de forma diferencial se usan tres electrodos por canal.

La finalidad de la etapa de amplificación es elevar la amplitud de la señal.

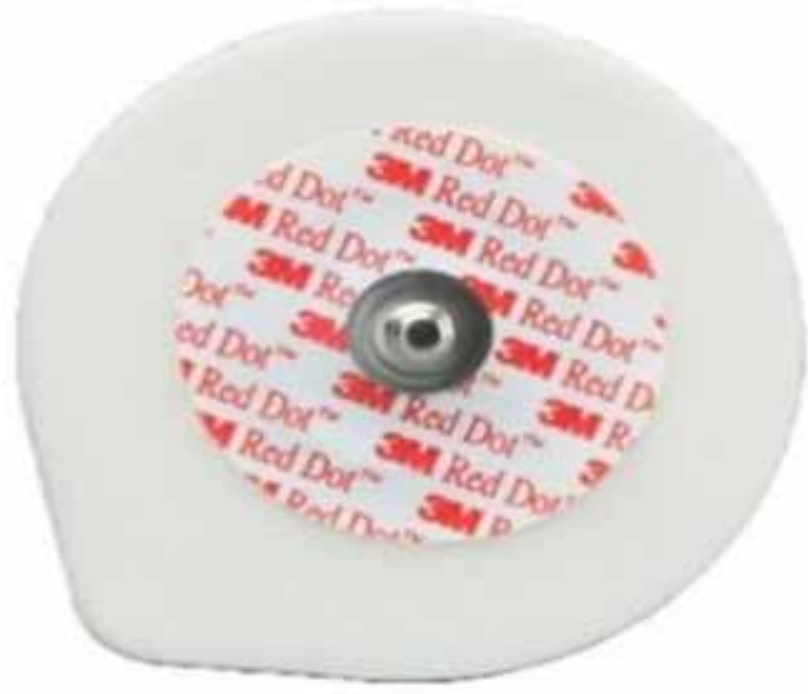

Figura 1. Electrodos cloruro de plata 3M. 


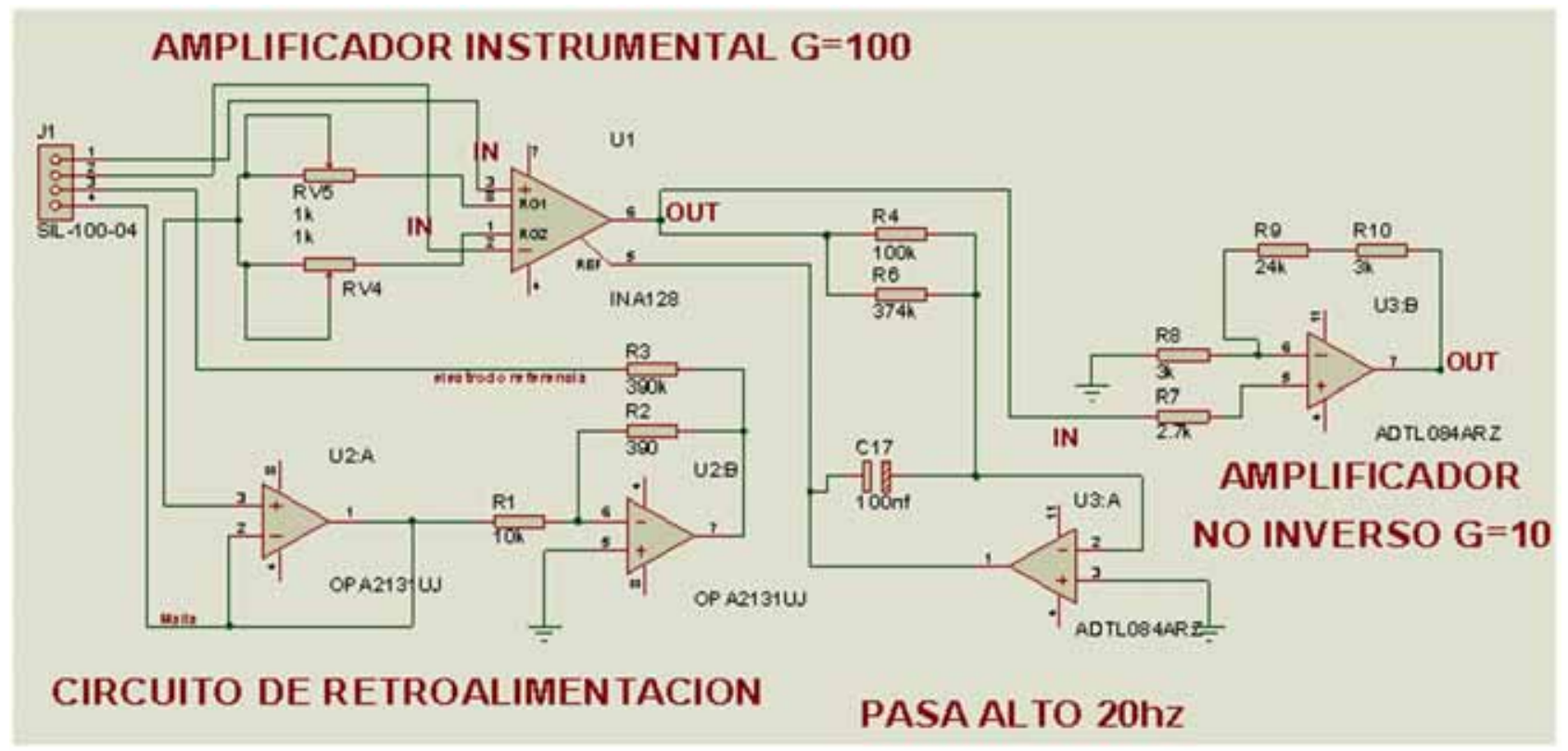

Figura 2. Etapa de adquisición y amplificación.

Primero se utiliza el amplificador de instrumentación INA128 con una ganancia de 50 . Se utiliza este amplificador por cumplir con las recomendaciones mínimas de la Sociedad Internacional de Electrofisiología y Kinesiología (ISEK).

Además se implementa el circuito de la pierna derecha del ECG (TI, 2005) calculada en la hoja de datos del amplificador de instrumentación, adaptándolo al EMG, circuito útil para evitar las corrientes de desbalance y compensar problemas de ruido de modo común en la entrada diferencial del amplificador de instrumentación. La modificación de este circuito consiste en cambiar la posición de los electrodos del ECG al EMG, al ubicar los electrodos RA y LA alrededor del músculo y el electrodo RL como electrodo de referencia. También cuenta con una salida de blindaje o de malla evitando corrientes de fuga de los cables de los electrodos.

A la salida del amplificador de instrumentación, se utiliza un lazo de realimentación autocero o de acoplamiento AC, válido como un filtro paso alto de 20 $\mathrm{Hz}$ (Pérez, 2007), frecuencia recomendada de estudios previos de trabajos realizados en detección y registro de señales mioeléctricas. Esta configuración es recomendada de la hoja de datos del amplificador de instrumentación, para obtener lecturas únicamente de señales mioeléctricas, y posee muy pocos elementos electrónicos para su montaje, obteniedo el mismo resultado de un filtro pasa alto Sallen-Key.
Posteriormente, se implementa después de la etapa de preamplificación, construida con amplificadores operaciones TL084 en configuración de amplificador no inversor de ganancia 10, con la finalidad de no desfasar la señal filtrada y alcanzar los niveles de voltajes óptimos y aceptables del convertidor analógico-digital interno en el microprocesador 16F877A.

El diagrama completo de la etapa de adquisición y amplificación, se puede observar en la Figura 2.

\subsection{Filtro Notch Fliege}

El filtro Notch Fliege (Carter, 2006) es útil para eliminar interferencias en señales de pequeñas amplitudes, éste tipo de filtro presenta la ventaja de poseer solo cuatro elementos para el ajuste de la frecuencia central y un cambio leve en el valor de los componentes no afecta la frecuencia de corte ni la atenuación del filtro, también se puede ajustar el factor de calidad de forma independiente; estas características no las poseen otros filtros Notch como por ejemplo el filtro Notch doble T.

La frecuencia de ruido de $60 \mathrm{~Hz}$ de la red eléctrica domiciliaria se atenúa implementando dos filtros Notch Fliege a $60 \mathrm{~Hz}$ en serie (Figura 3), con un factor de calidad Q de 38, valor escogido debido a que se presentaba una menor afectación en la respuesta del filtro ante variaciones en sus componentes. 


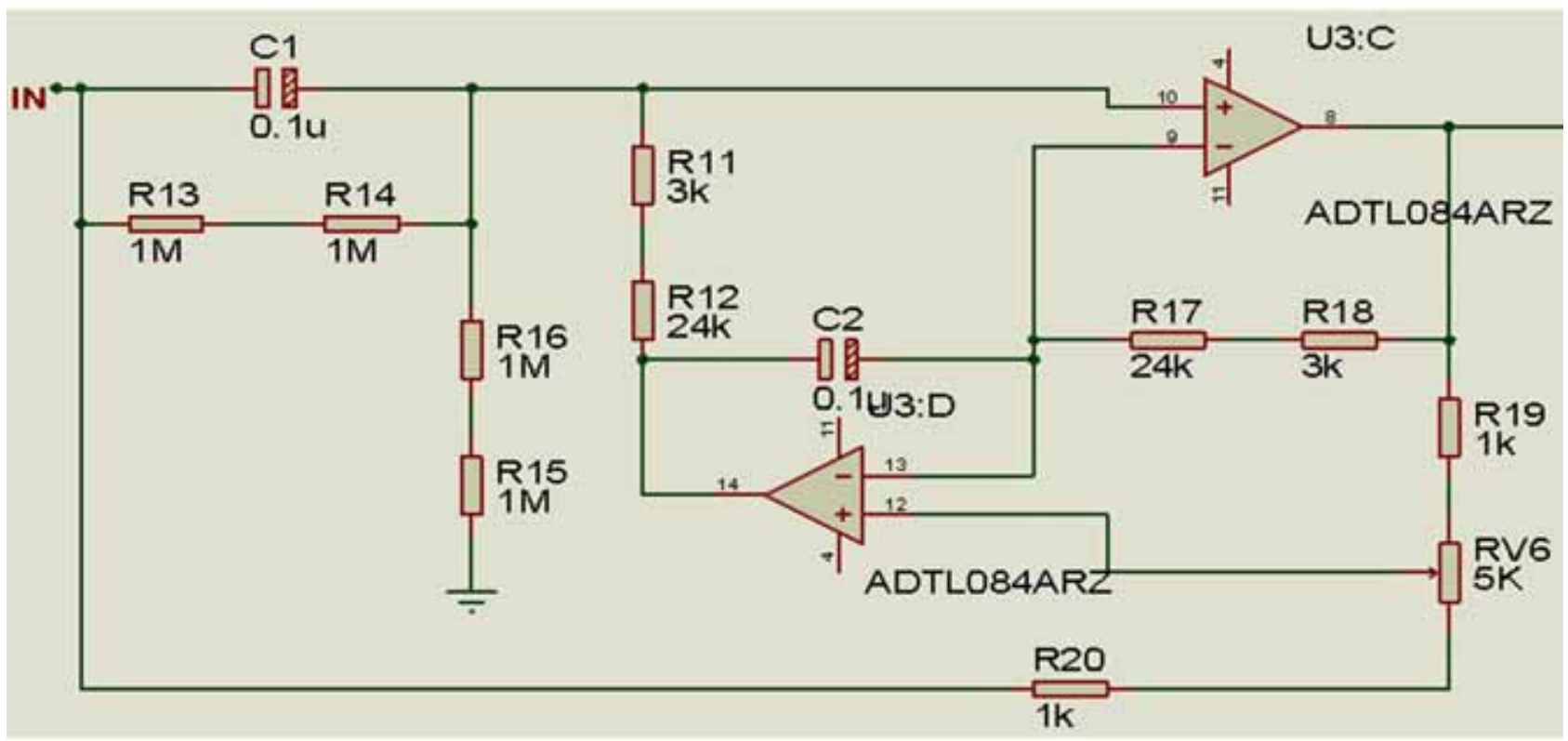

Figura 3. Filtro Notch.

\subsection{Filtro paso bajo}

Para eliminar el ruido de alta frecuencia en la señal EMG y eliminar el aliasing, es necesario filtrar señal. Se implementó un filtro pasa bajas de $500 \mathrm{~Hz}$ SallenKey tipo Bessel de cuarto orden (Figura 4).

\subsection{Sumador no inversor y limitador}

Los potenciales de acción de los músculos en esta etapa tienen valores desde $-2.5 \mathrm{~V}$ a $2.5 \mathrm{~V}$, obtenidos de las etapas de amplificación y filtrado previo. Debido a que el conversor análogo-digital solo recibe señales de $0 \mathrm{~V}$ a $5 \mathrm{~V}$, es necesario sumarle a la señal un valor de $2.5 \mathrm{~V}$, esto se realiza por medio de un sumador no inversor
(Figura 5), además en esta etapa existe un limitador, el cual evita daños en el convertidor análogo-digital interndel microcontrolador 16F877A, al solo aceptar valores de voltaje entre $-0.5 \mathrm{~V}$ a $5.5 \mathrm{~V}$.

\subsection{Comunicación serial y microcontrolador 16F877A}

Se utilizó el microcontrolador 16F877A con protocolo de comunicación serial asíncrono simplex, para enviar las tramas desde el EMG al receptor XBee Explorer, con el fin de reducir los retardos y la perdida de datos del censando de los cuatro canales; el proceso de habilitación de la USART se efectúa a través de los registros SPBRG el cual maneja una

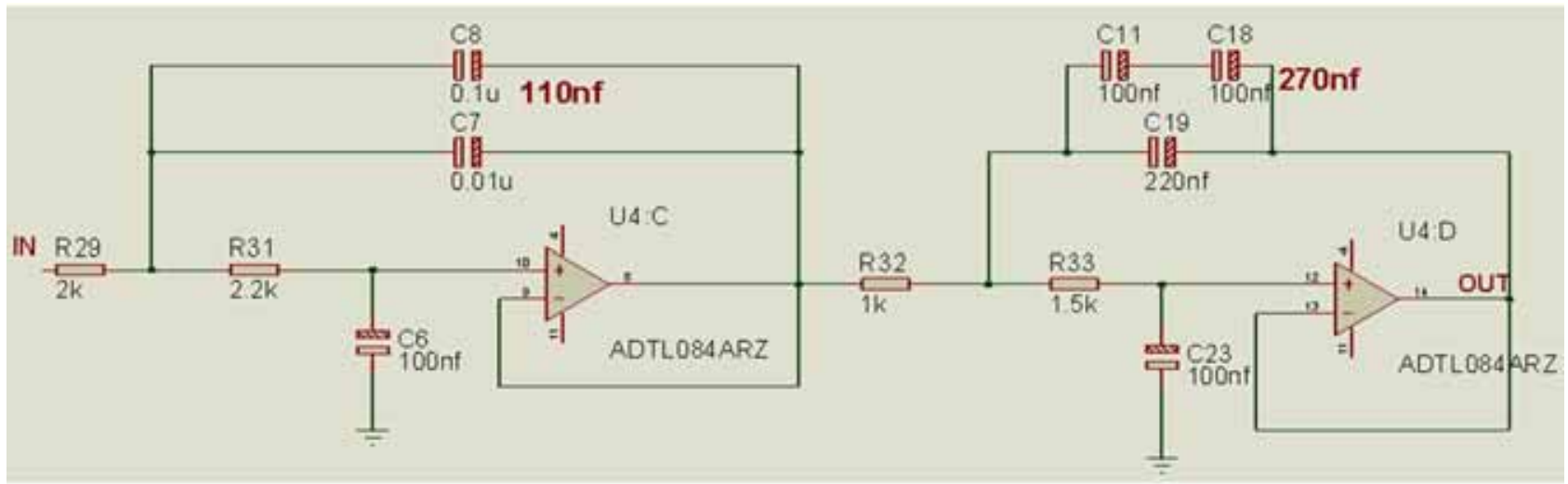

Figura 4. Filtro paso bajo. 


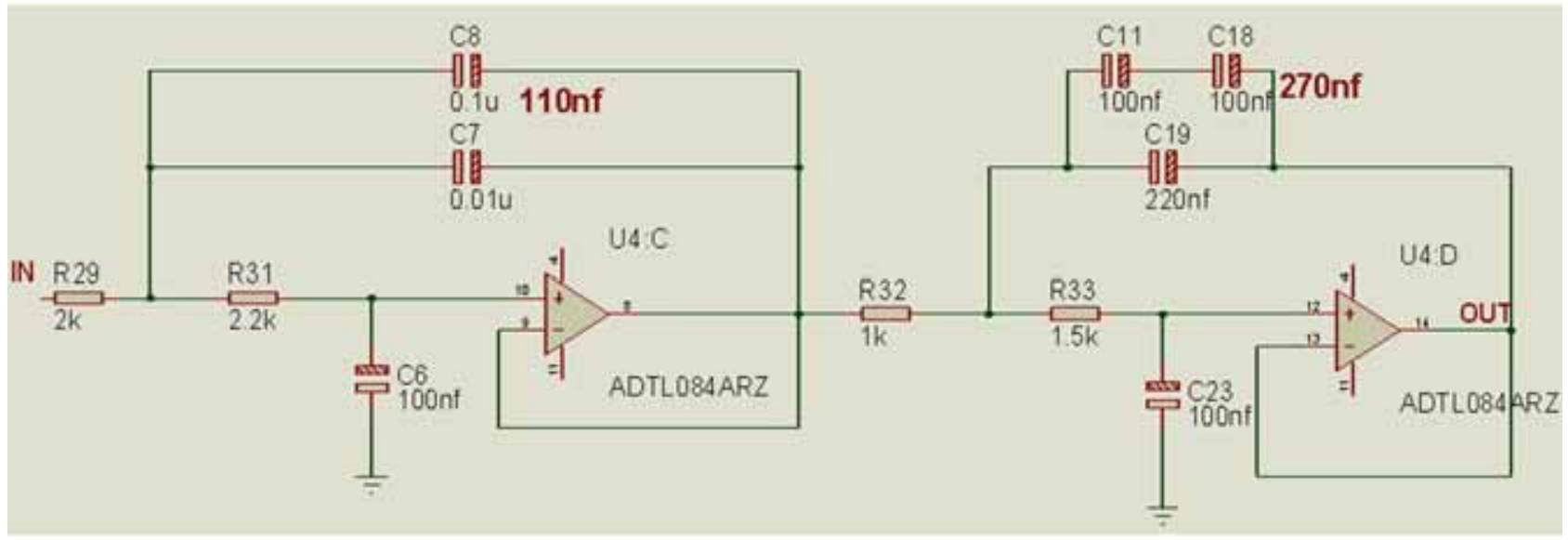

Figura 5. Sumador no inversor y limitador.

tasa de 9600 baudios, además se usan los registros TXSTA y RCSTA para controlar el estado de la transmisión y recepción; conjuntamente se emplean los registros TXREG y RCREG para la transmisión y recepción de los datos.

El firmware del microcontrolador 16F877A (Figura 6) está diseñado para enviar una trama completa de diez bytes, de los cuales ocho bytes son para los datos de los cuatro canales, cada canal está compuesto por 8 bits del nibble superior y 2 bits del nibble inferior.

Para la comunicación inalámbrica entre el EMGs y el computador, se utilizaron módulos XBee, que usan protocolo IEEE 802.15.4, el cual se ubica en la capa física con modulación (2.4 GhZ 16-QAM, 915 Mhz y 868 Mhz (Figura 6); Otro aspecto a destacar de estos dispositivos es la fácil configuración del enlace entre los módulos.
La configuración del enlace entre los dos módulos XBEE es de 9600 bps, 8 bits y sin paridad, suficiente para poder enviar las señales adquiridas y visualizarlas en tiempo real.

\subsection{Alimentación}

La alimentación de los canales SMD depende de un convertidor DC-DC bipolar Reliability 3W12R12-12, el cual se encarga de entregar $\pm 12 \mathrm{~V}$ a cada canal, teniendo una alimentación constante; además se utilizó un regulador LM7805 para alimentar con 5V el microcontrolador 16F877A, de la misma manera se usó el LM317 para obtener los 3.3V necesarios para la alimentación del módulo XBee. En la Figura 6 se muestra la configuración de la alimentación de los cuatro canales, el microcontrolador 16F877A y del módulo XBee.

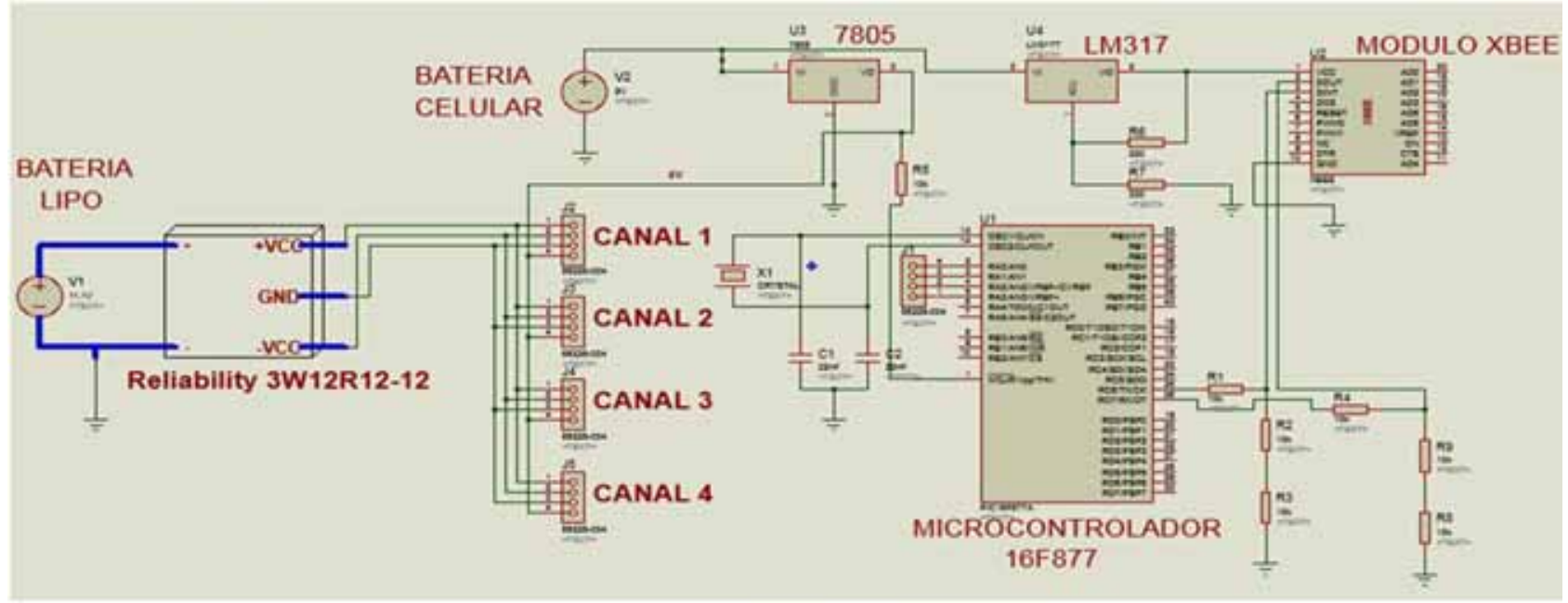

Figura 6. Alimentación, XBee, microcontrolador 16F877A. 


\subsection{Decodificación de la trama en labVIEW}

Se utiliza LabVIEW por ser un lenguaje de programación gráfica denominado lenguaje $\mathrm{G}$, además de poseer herramientas para el procesamiento de las señales, tales como análisis Wavelet.

Los datos en la trama serial se extraen de tal manera que las cuatro señales conserven sus características en precisión y resolución, necesarias para procesar cada una de las señales en tiempo real.

Los canales cuentan con un total de 8 string para los 4 canales y una resolución de 10 bits. Además a la trama se le agregaron dos identificadores de cabeceras para identificar y comprobar la llegada de la trama en LabVIEW de forma confiable, estableciendo una comunicación simplex serial RS232 (Figura 7).

Para detección de identificadores de trama (Figura 8), se decodifica a través de un array en LabVIEW, optimizando la velocidad de procesamiento y optimizando las muestras en término de tiempo.

Después se detectan, separan, concatenan y transforman las parejas de bytes correspondientes de cada canal, obteniendo la resolución de 10 bits por canal; para visualizar las cuatro señales electromiográficas, a continuación se utiliza un tab control para separar, activar y desactivar la adquisición de las señales electromiográficas (Figura 9) de la transformada de wavelet continua. Cada canal se hace independiente al usar un Case Structure, además se emplea un file patch en la creación de archivos bloc de notas (txt) con la función de grabar y leer los datos del canal correspondiente.

Ya adquiridas y acondicionadas las cuatro señales electromiográficas en el programa LabVIEW, se aplica la transformada wavelet continua (Silva, 2005); en la Figura 10, se puede observar la programación alambre de la Transformada Wavelet Continua.

\section{Resultados}

En la Figura 11 se muestra una señal electromiografica de un biceps en el dominio del tiempo, con un peso variable desde $2 \mathrm{Kg}$ a $7 \mathrm{Kg}$ con un aumento de $1 \mathrm{Kg}$. La señal obtenida es típica de una señal electromiográfica de una persona con condicion física normal. En la Figura 12 se observa la anterior señal electromiográfica procesada con la transformada Wavelet continua con las wavelets madres Db2 y Db4, a una escala de 32.

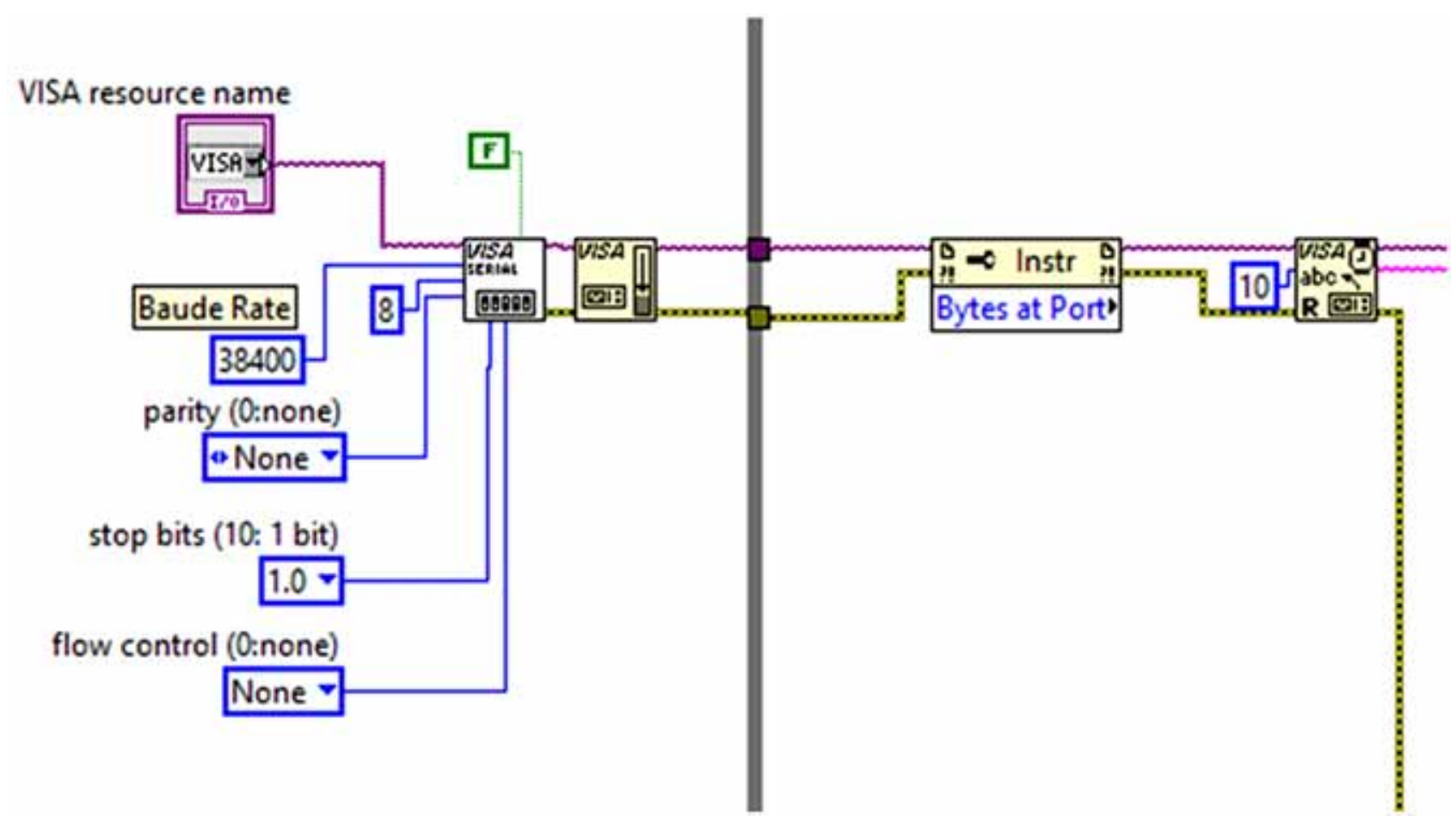

Figura 7. Comunicación simplex serial RS232. 


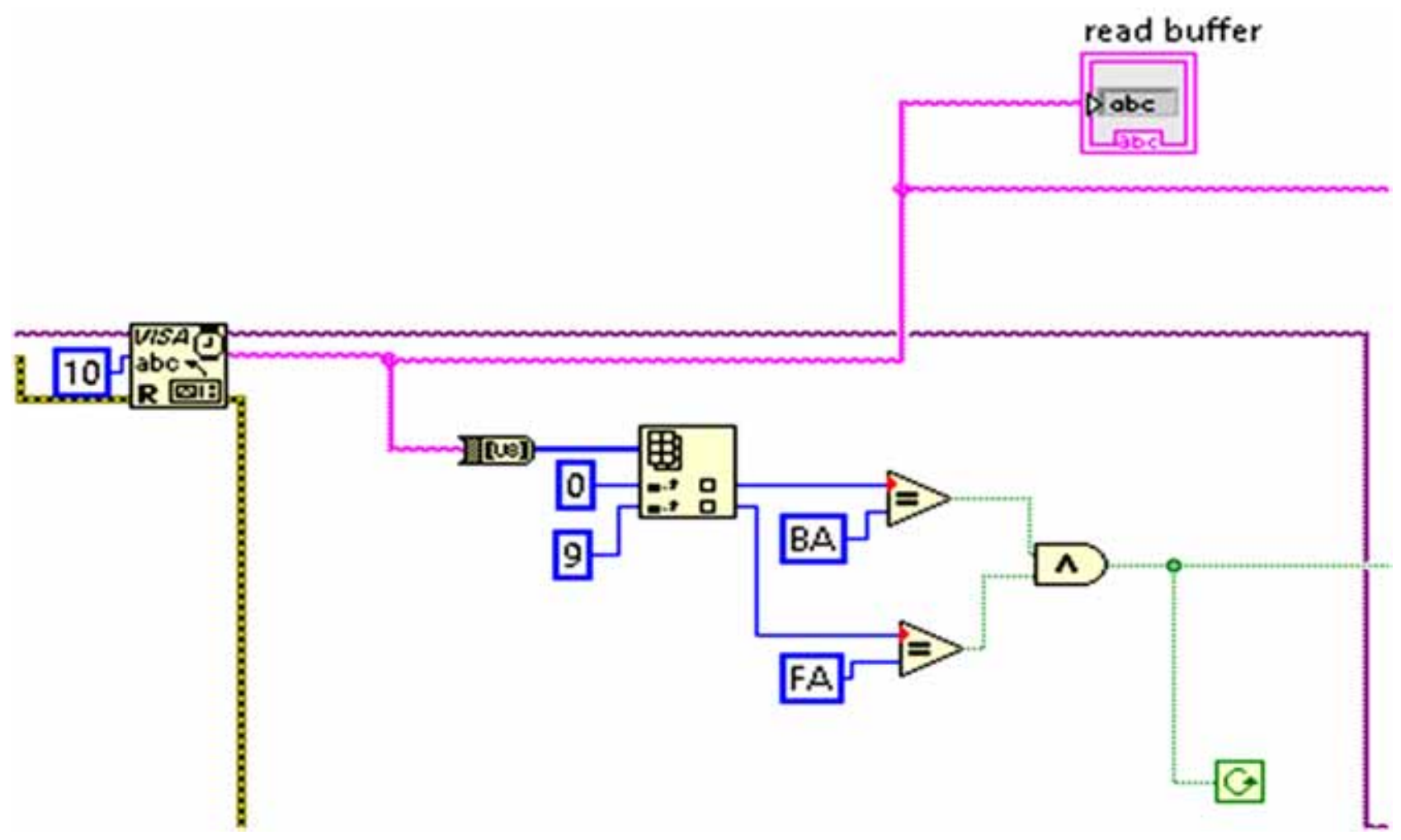

Figura 8. Detección de identificadores de trama.

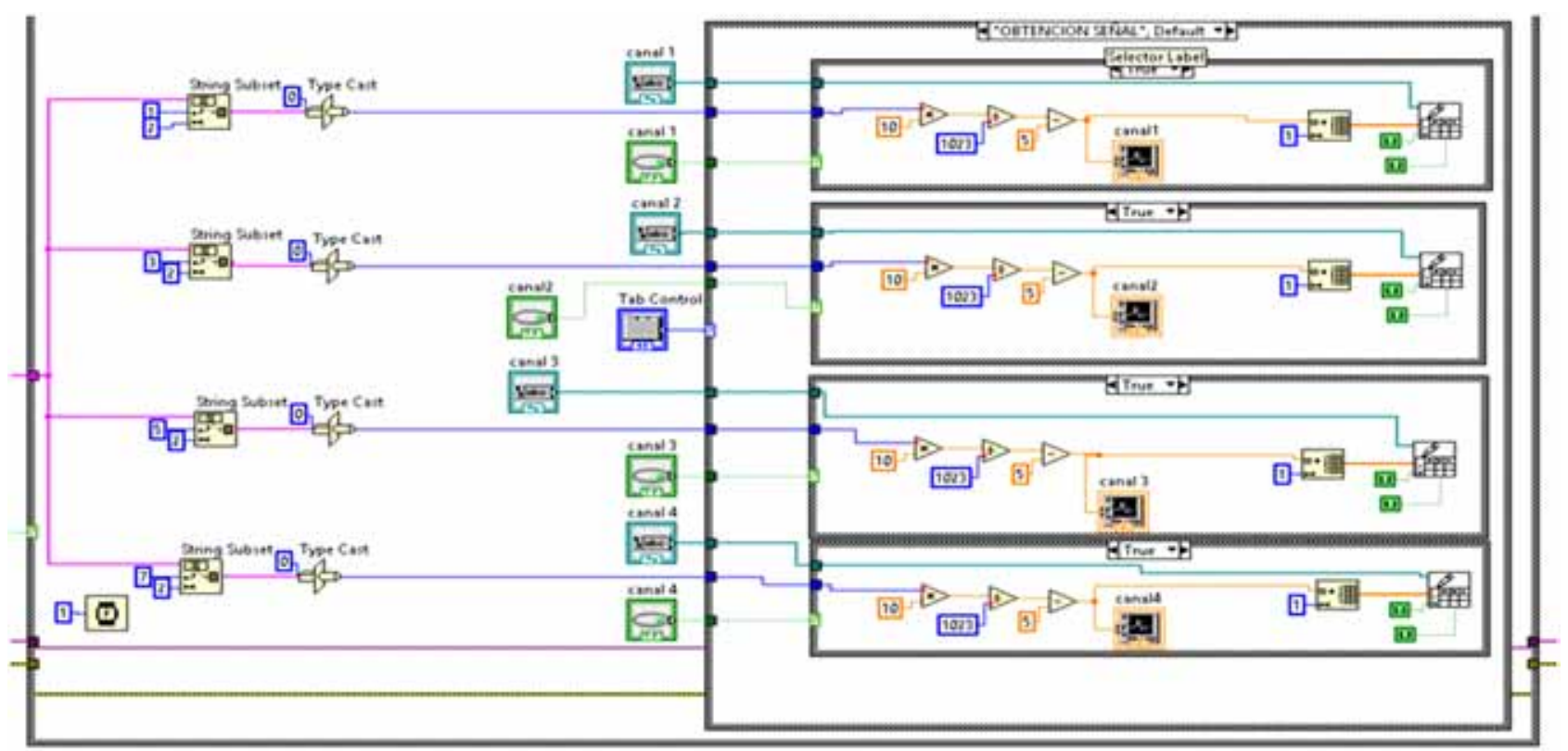

Figura 9. Adquisición de los 4 canales.

A continuación en las Tablas 1, 2, 3 y 4 se muestran los resultados de los diez sujetos de prueba al realizar las flexiones de bíceps, flexiones de brazo, flexiones de cuclillas y flexiones de pierna alternada. Cada prueba realizada es procesada con las wavelets madres Db4 y Db2, cada una con escalas de 32 y 250, para comparar el efecto de predominancia, concentración de escala y efectividad entre la predominancia real y la obtenida por CWT de cada sujeto de prueba. 


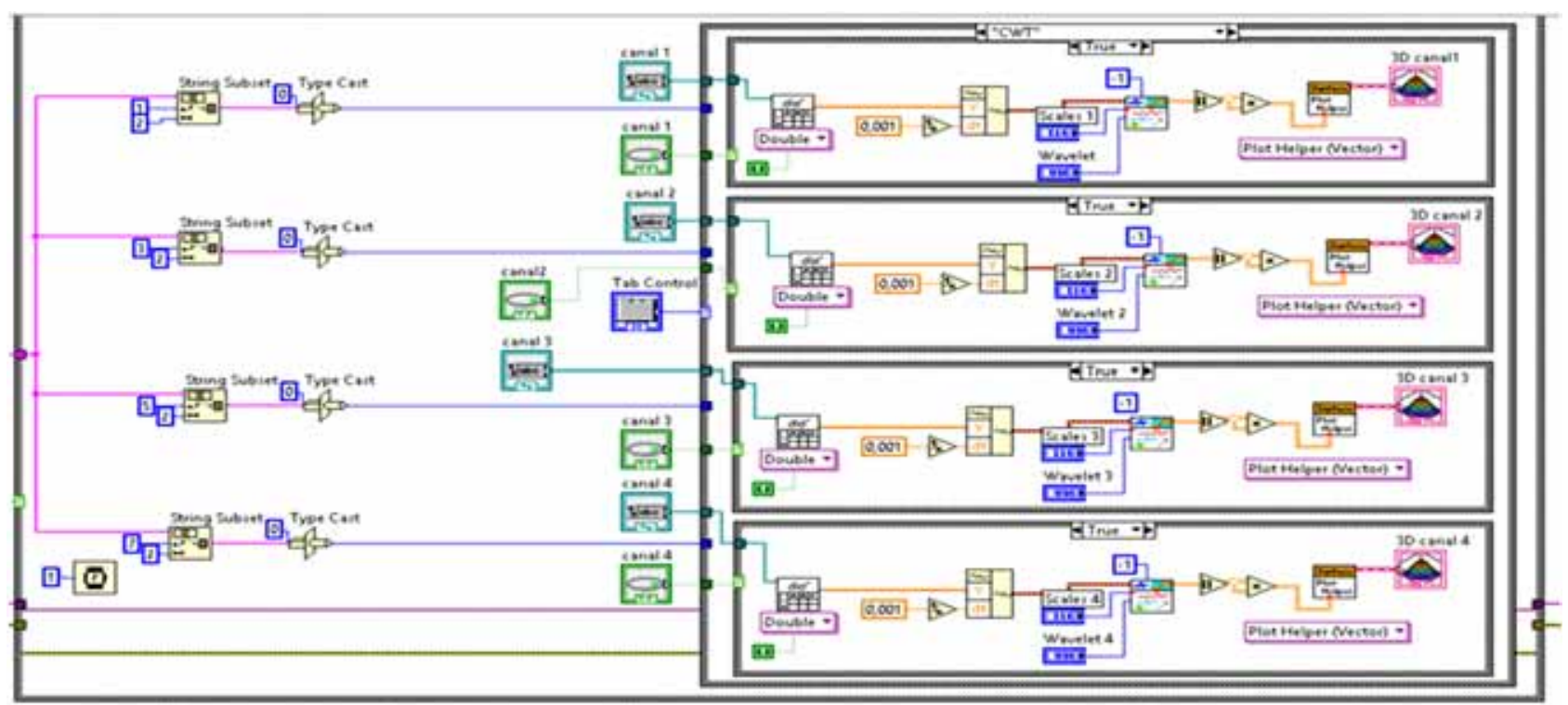

Figura 10. Transformada Wavelet Continúa de los 4 canales.

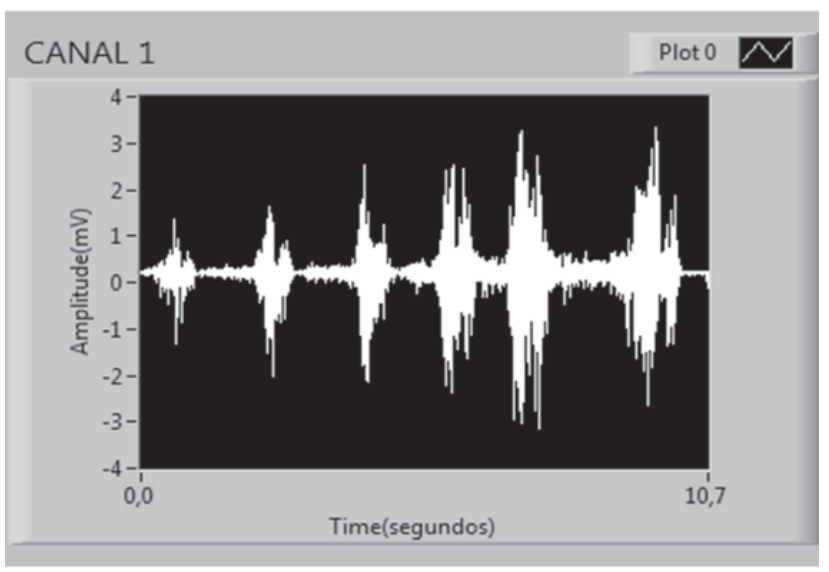

Figura 11. Señales obtenidas de los bíceps.
Realizando la comparación entre la escala de 250, con respecto a una escala de 32, se observa que al utilizar una escala mayor en este caso aumentan los valores del eje de la amplitud, amplitudes concentradas en escalas altas con la presencia de frecuencias muy bajas, datos no concordantes con la realidad.

Usando la wavelet madre Db4, se consiguió un mejor resultado, por el parecido de la wavelet madre $\mathrm{Db} 4 \mathrm{a}$ un potencial de acción de unidad motora y el hecho de que la Transformada Wavelet realiza una convolución de la wavelet madre Db4 con la señal a procesar. Por lo tanto, los valores similares de la señal en estudio

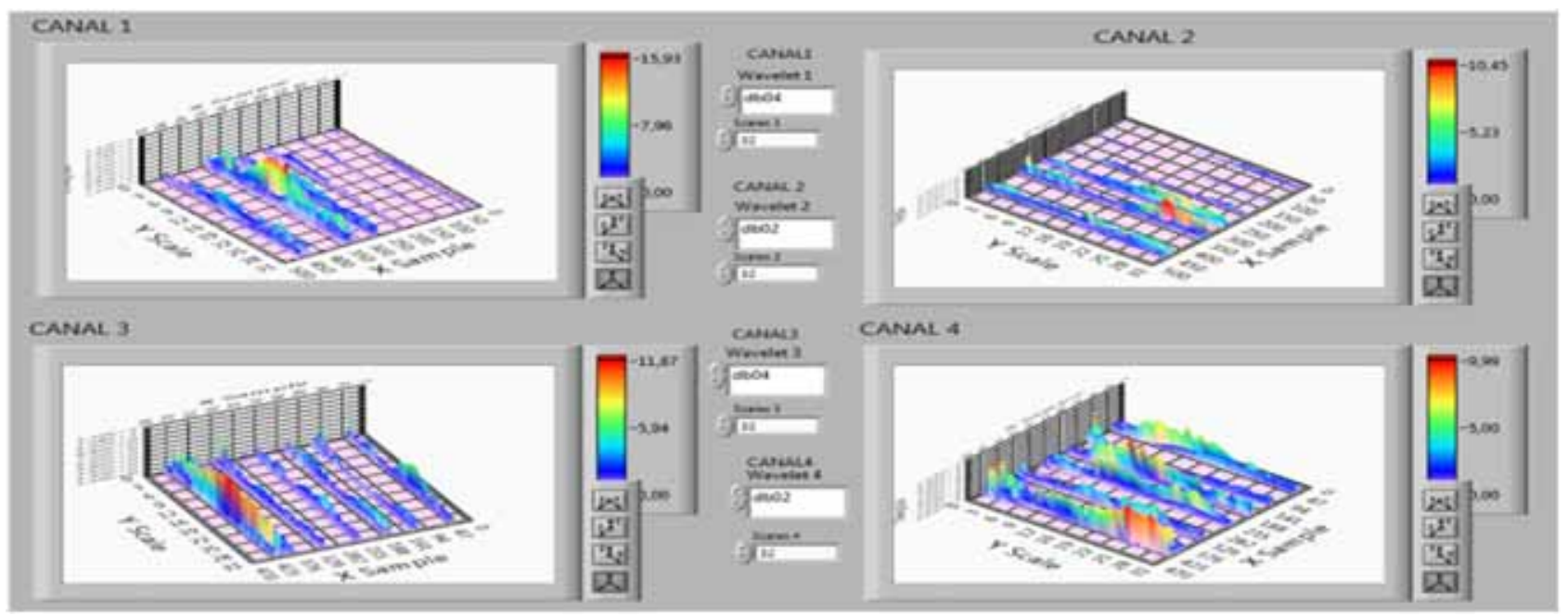

Figura 12. Escalograma flexión de bíceps sujeto1. Canal1 y canal 2 brazo derecho, canal 3 y canal 4 brazo izquierdo. 
Tabla 1. Flexiones de biceps.

\begin{tabular}{|c|c|c|c|c|c|c|c|c|c|c|c|c|c|c|}
\hline \multicolumn{15}{|c|}{ Flexiones de biceps } \\
\hline \multirow{3}{*}{$\begin{array}{c}\text { Wavelet madre } \\
\text { Escala }\end{array}$} & \multicolumn{7}{|c|}{ Db4 } & \multicolumn{7}{|c|}{ Db2 } \\
\hline & \multicolumn{3}{|c|}{32} & \multicolumn{3}{|c|}{250} & \multirow[b]{2}{*}{ REAL } & \multicolumn{3}{|c|}{32} & \multicolumn{3}{|c|}{250} & \multirow[b]{2}{*}{ REAL } \\
\hline & Der & Izq & EC & Der & Izq & EC & & Der & Izq & $\mathrm{EC}$ & Der & $\mathrm{lzq}$ & $\mathrm{EC}$ & \\
\hline $\mathrm{HI}$ & $\mathrm{X}$ & & B & $X$ & & $A$ & D & $X$ & & B & $\mathrm{X}$ & & $\mathrm{A}$ & $\bar{D}$ \\
\hline $\mathrm{H} 2$ & $\mathrm{X}$ & & B & & $\bar{x}$ & A & $\mathrm{D}$ & & X & 1 & $\mathrm{X}$ & & $\bar{A}$ & $\mathrm{D}$ \\
\hline $\mathrm{H} 3$ & $\mathrm{X}$ & & 1 & & $\mathrm{X}$ & A & IZ & & $\mathrm{X}$ & 1 & & $X$ & $\mathrm{~A}$ & $\mathrm{IZ}$ \\
\hline $\mathrm{H} 4$ & $\mathrm{X}$ & & $\mathrm{I}$ & 3 & & A & $\mathrm{D}$ & $\mathrm{X}$ & & $\mathrm{I}$ & & & $\mathrm{A}$ & $\mathrm{D}$ \\
\hline $\mathrm{H} 5$ & $\mathrm{X}$ & & B & & $\mathrm{X}$ & A & $\mathrm{D}$ & $\mathrm{X}$ & & B & & & $A$ & $\mathrm{D}$ \\
\hline H6 & $\mathrm{X}$ & & B & & 8 & $\mathrm{~A}$ & D & $\mathrm{X}$ & & 1 & $X$ & & A & D \\
\hline $\mathrm{H7}$ & & $\mathrm{X}$ & 1 & & $\mathrm{X}$ & $\mathrm{A}$ & $\mathrm{K}$ & $\mathrm{X}$ & & 1 & & & $\mathrm{~A}$ & 12 \\
\hline MI & $\mathrm{O}$ & & 1 & & 10 & A & D & 0 & & 1 & & & $\mathrm{~A}$ & D \\
\hline $\mathrm{M} 2$ & $\mathrm{O}$ & & I & 0 & & $\mathrm{~A}$ & $\mathrm{D}$ & $\mathrm{O}$ & & 1 & $\mathrm{O}$ & & $\mathrm{A}$ & $\mathrm{D}$ \\
\hline M3 & & $\mathrm{O}$ & I & & 0 & $A$ & IZ & & $\mathrm{O}$ & A & & & $A$ & IZ \\
\hline$\%$ efectivo & \multicolumn{2}{|l|}{90} & & \multicolumn{2}{|l|}{60} & & & \multicolumn{2}{|l|}{80} & & \multicolumn{2}{|l|}{50} & & \\
\hline $\begin{array}{l}\mathrm{H}=\text { Hombre } \\
\mathrm{Ec}=\text { Escala Conce }\end{array}$ & $\begin{array}{l}=\text { Muj } \\
\text { ntrada }\end{array}$ & & $\begin{array}{l}\mathrm{B}=1 \\
\mathrm{D}=\end{array}$ & $\begin{array}{l}\text { 3aja } \\
\text { Extrem }\end{array}$ & dad D & recha & $\begin{array}{l}\mathrm{I}=\mathrm{In} \\
\mathrm{I} z=\mathrm{I}\end{array}$ & $\begin{array}{l}\text { termed } \\
\text { xtrem }\end{array}$ & dad Iz & uierd: & & & ta & \\
\hline
\end{tabular}

Tabla 2. Resultado efectividad predominancia entre CWT y real.

\begin{tabular}{|c|c|c|c|c|c|c|c|c|c|c|c|c|c|c|}
\hline \multicolumn{15}{|c|}{ Flexiones de brazos } \\
\hline \multirow{3}{*}{$\begin{array}{c}\text { Wavelet madre } \\
\text { Escala }\end{array}$} & \multicolumn{7}{|c|}{ Db4 } & \multicolumn{7}{|c|}{ Db2 } \\
\hline & \multicolumn{3}{|c|}{32} & \multicolumn{3}{|c|}{250} & \multirow[b]{2}{*}{ REAL } & \multicolumn{3}{|c|}{32} & \multicolumn{3}{|c|}{250} & \multirow[b]{2}{*}{ REAI } \\
\hline & Der & Izq & $\mathrm{EC}$ & Der: & $1 z q$ & $\mathrm{EC}$ & & Der & Izq & $\mathrm{EC}$ & Der & Irq & $E C$ & \\
\hline $\mathrm{HI}$ & & $\mathrm{X}$ & 1 & & $X$ & $\mathrm{~A}$ & D & & $x$ & 1 & & & $\mathrm{~A}$ & $\mathrm{D}$ \\
\hline $\mathrm{H} 2$ & & $\mathrm{X}$ & 1 & & $\bar{Y}$ & $\mathrm{~A}$ & D & & $\mathrm{X}$ & I & & & A & $\mathrm{D}$ \\
\hline $\mathrm{H} 3$ & & $\mathrm{X}$ & 1 & & $\mathrm{X}$ & A & IZ & & $\mathrm{X}$ & 1 & & $X$ & A & 12 \\
\hline $\mathrm{H} 4$ & $\mathrm{X}$ & & I & $\mathrm{X}$ & & A & D & $\mathrm{X}$ & & 1 & $\mathrm{X}$ & & A & $\mathrm{D}$ \\
\hline H5 & $\mathrm{X}$ & & B & & 8 & A & D & $\mathrm{X}$ & & $\mathrm{B}$ & $X$ & & A. & D \\
\hline H6 & $\mathrm{X}$ & & 1 & & +4 & A & $\mathrm{D}$ & $\mathrm{X}$ & & 1 & & & $\mathrm{~A}$ & $\mathrm{D}$ \\
\hline $\mathrm{H7}$ & & $\mathrm{X}$ & 1 & & $X$ & A & IZ & & $\mathrm{X}$ & 1 & & $\mathrm{X}$ & $\mathrm{A}$ & IZ \\
\hline M1 & $\mathrm{O}$ & & I & 0 & & $A$ & $\mathrm{D}$ & $\mathrm{O}$ & & I & $\mathrm{O}$ & & A & $\mathrm{D}$ \\
\hline M2 & $\mathrm{O}$ & & I & & 0 & $\mathrm{~A}$ & $\mathrm{D}$ & & 0 & I & & $\overline{\text { (11) }}$ & $A$ & $\mathrm{D}$ \\
\hline M3 & & $\mathrm{O}$ & 1 & & 0 & A & IZ & & 0 & A & & 0 & $A$ & $1 Z$ \\
\hline$\%$ efectivo & \multicolumn{2}{|l|}{80} & & \multicolumn{2}{|l|}{60} & & & \multicolumn{2}{|l|}{70} & & \multicolumn{2}{|l|}{60} & & \\
\hline $\begin{array}{l}\mathrm{H}=\text { Hombre } \\
\mathrm{Ec}=\text { Escala Concer }\end{array}$ & $\begin{array}{l}=\text { Muj } \\
\text { trada }\end{array}$ & & $\begin{array}{l}B=1 \\
D=1\end{array}$ & $\begin{array}{l}\text { aja } \\
\text { xtremi }\end{array}$ & lad De & echa & $\begin{array}{l}\mathrm{I}=\mathrm{In} t \\
\mathrm{I} z=\mathrm{E}\end{array}$ & $\begin{array}{l}\text { ermedi } \\
\text { xtremic }\end{array}$ & $\mathrm{Izq}$ & ierda & & $A=$ & & \\
\hline
\end{tabular}


Tabla 3. Flexiones de cuclillas.

\begin{tabular}{|c|c|c|c|c|c|c|c|c|c|c|c|c|c|c|}
\hline \multicolumn{15}{|c|}{ Cuclillas } \\
\hline \multirow{3}{*}{$\begin{array}{c}\text { Wavelet madre } \\
\text { Escala }\end{array}$} & \multicolumn{7}{|c|}{ Db4 } & \multicolumn{7}{|c|}{ Db2 } \\
\hline & \multicolumn{3}{|c|}{32} & \multicolumn{3}{|c|}{250} & \multirow[b]{2}{*}{ REAl } & \multicolumn{3}{|c|}{32} & \multicolumn{3}{|c|}{250} & \multirow[b]{2}{*}{ RFAI } \\
\hline & Der & $\mathrm{Izq}$ & $\mathrm{EC}$ & Der & 129 & $\mathrm{EC}$ & & Der & Izq & $\mathrm{EC}$ & Der & 149 & $E C$ & \\
\hline $\mathrm{HI}$ & $\mathrm{X}$ & & B & & $y$ & A & $D$ & $\mathrm{x}$ & & B & & & A & D) \\
\hline $\mathrm{H} 2$ & & $\mathrm{X}$ & B & $\mathrm{X}$ & & $A$ & D & & $\mathrm{X}$ & B & & 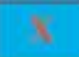 & A & $\mathrm{D}$ \\
\hline $\mathrm{H} 3$ & & $\mathrm{X}$ & I & & $X$ & A & 17 & & $X$ & 1 & & $x$ & A & 16 \\
\hline $\mathrm{H} 4$ & $\mathrm{X}$ & & 1 & $X$ & & A & D) & $X$ & & 1 & $x$ & & A & 1) \\
\hline H5 & $\mathrm{X}$ & & B & & W & A & $\mathrm{B}$ & $x$ & & B & & 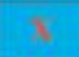 & A & D) \\
\hline$H 6$ & $\mathrm{X}$ & & 1 & $X$ & & $A$ & D) & $\mathrm{X}$ & & 1 & $x$ & & $\Lambda$ & 1) \\
\hline $\mathrm{H7}$ & & $\mathrm{X}$ & B & & $X$ & $A$ & IZ & & $\mathrm{X}$ & B & & $x$ & $A$ & 12 \\
\hline MI & O & & 1 & 0 & & $A$ & D & 0 & & 1 & 0 & & $\Lambda$ & D \\
\hline M2 & $\mathrm{O}$ & & I & & CI & $\Lambda$ & D & 0 & & I & & 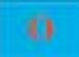 & $\Lambda$ & D \\
\hline M3: & & $\mathrm{O}$ & I & & 0 & $\Lambda$ & 12 & & 0 & I & & 0 & $\Lambda$ & IR \\
\hline$\%$ efectivo & \multicolumn{2}{|l|}{90} & & \multicolumn{2}{|l|}{50} & & & \multicolumn{2}{|l|}{80} & & \multicolumn{2}{|l|}{60} & & \\
\hline $\begin{array}{l}\mathrm{H}=\text { Hombre } \\
\mathrm{Ec}=\text { Escala Conce }\end{array}$ & rada & & & $\begin{array}{l}\text { Baja } \\
\text { Extren }\end{array}$ & idad & erech & $\begin{array}{l}\mathrm{I}= \\
\mathrm{I} z=\end{array}$ & $\begin{array}{l}\text { Interm } \\
\text { Extrer }\end{array}$ & $\begin{array}{l}\text { dia } \\
\text { idad }\end{array}$ & zquierda & & $=\mathrm{A}$ & & \\
\hline
\end{tabular}

Tabla 4. Flexiones de piernas alternada.

\section{Flexiones de pierna alternada}

\begin{tabular}{|c|c|c|c|c|c|c|c|c|c|c|c|c|c|c|}
\hline Wavelet madre & \multicolumn{7}{|c|}{ Db4 } & \multicolumn{7}{|c|}{$\mathrm{Db} 2$} \\
\hline \multirow[t]{2}{*}{ Escala } & \multicolumn{3}{|c|}{32} & \multicolumn{3}{|c|}{250} & & \multicolumn{3}{|c|}{32} & \multicolumn{3}{|c|}{2811} & \\
\hline & Der & $\mathrm{lzq}$ & $\mathrm{EC}$ & Der & 129 & $\mathrm{EC}$ & & Der & lzq & $\mathrm{EC}$ & Der & 179 & $\mathrm{HC}^{\circ}$ & \\
\hline $\mathrm{HI}$ & & $\mathbf{X}$ & B & & X & A & $\mathrm{D}$ & & $\mathrm{X}$ & B & & 5 & A & $\mathrm{D}$ \\
\hline $\mathrm{H2}$ & & $\mathrm{X}$ & B & & $\mathrm{X}$ & A & D & & $x$ & B & $x$ & & A & D) \\
\hline $\mathrm{H} 3$ & & $\mathrm{X}$ & I & & $\mathrm{X}$ & $\Lambda$ & W. & & $X$ & 1 & & $x$ & $\lambda$ & B \\
\hline $\mathrm{H} 4$ & $\mathrm{x}$ & & I & & $x$ & $A$ & $\mathrm{D}$ & $\mathrm{X}$ & & I & $\mathrm{X}$ & & A & D \\
\hline H5 & $\mathrm{X}$ & & B & $x$ & & A & D) & $\mathrm{X}$ & & I & & & $\Lambda$ & () \\
\hline 116 & $\mathrm{X}$ & & I & & 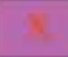 & A & D & $\mathrm{X}$ & & 1 & & & $A$ & D) \\
\hline $\mathrm{H7}$ & & $\mathrm{X}$ & I & & $X$ & $\Lambda$ & K & & $\mathrm{X}$ & 1 & & $x$ & $A$ & B \\
\hline M1 & O & & I & 0 & & A & D & 0 & & I & & & $A$ & $\mathrm{D}$ \\
\hline $\mathrm{M} 2$ & 0 & & 1 & & 31 & A & D & & 0 & 1 & 0 & & $A$ & D \\
\hline $\mathrm{M}$ & & $\mathrm{O}$ & 1 & 1) & & A & 12 & & 0 & A & & & $\Lambda$ & 17 \\
\hline$\%$ efectivo & \multicolumn{2}{|l|}{90} & & \multicolumn{2}{|l|}{50} & & & \multicolumn{2}{|l|}{70} & & \multicolumn{2}{|l|}{50} & & \\
\hline $\begin{array}{l}\mathrm{H}=\text { Hombre } \mathrm{M} \\
\mathrm{Ec}=\text { Escala Concer }\end{array}$ & $\begin{array}{l}\text { Muje: } \\
\text { ada }\end{array}$ & & $\begin{array}{l}B=B \\
=E x\end{array}$ & remida & Dere & & $\begin{array}{l}=\operatorname{Int} \\
z=E\end{array}$ & $\begin{array}{l}\text { media } \\
\text { remid: }\end{array}$ & Izqu & rda & & $\mathrm{Al}$ & & \\
\hline
\end{tabular}


con la wavelet madre Db4 dan los valores de coeficientes más altos.

Al aumentar el potencial de acción de las unidades motoras y la frecuencia, tambien aumentan los valores del espectro de la señal electromiográfica de la Transformada Wavelet Continua, pero se concentra en escalas bajas.

La Figura 13 muestra la comparación de efectividad de la predominancia entre CWT y la predominancia real de los sujetos de las Tablas 1, 2, 3 y 4.

En la Figura 13, aplicando la wavelet madre Db4 con una escala de 32 se tiene una efectividad del $80 \%$ al $90 \%$, como segunda opción está el uso de la wavelet madre Db2 con una escala de 32 con una efectividad del $70 \%$ al $80 \%$, las barras menos efectivas son las Db4 y Db2 con una escala de 250, por medio de esta gráfica se confirma la efectividad de la escala 32, en comparación de la escala 250.

Utilizando la wavelet madre Db4 con una escala de 32, se observan frecuencias altas en escalas bajas y frecuencias intermedias en escalas intermedias con una distribución uniforme de estas frecuencias, concordante a las frecuencias en escalas esperadas en teoría, al ser esfuerzos físicos de los sujetos de pruebas por ser mayoría de hombres. Con la wavelet madre Db2 con una escala de 32, las frecuencias altas e intermedias se ubican la mayoría en escalas intermedias, algunas en escalas bajas, también se encuentran frecuencias en escalas altas esta información todavía tiene datos relevantes, aunque tiene un mayor margen de error en comparación a la wavelet madre Db4. Al utilizar la escala 250 sin importar el tipo de wavelet madre a usar, siempre se van a concentrar las frecuencias en escalas altas, información muy distinta a la realidad, algo muy poco útil para obtener datos.

La CWT cuenta con un gran número de familias de wavelets madre, que permite el análisis de una señal con funciones que resultan en una mejor obtención de la representación. Por otro lado, de modo que pueda conseguir una buena resolución de la señal, es necesario seleccionar una familia wavelet apropiada. En algunos casos, de acuerdo con la característica de la señal es necesaria una investigación para determinar la mejor wavelet madre.

Las señales electromiográficas obtenidas de los sujetos tienen una representación en el dominio del tiempo,

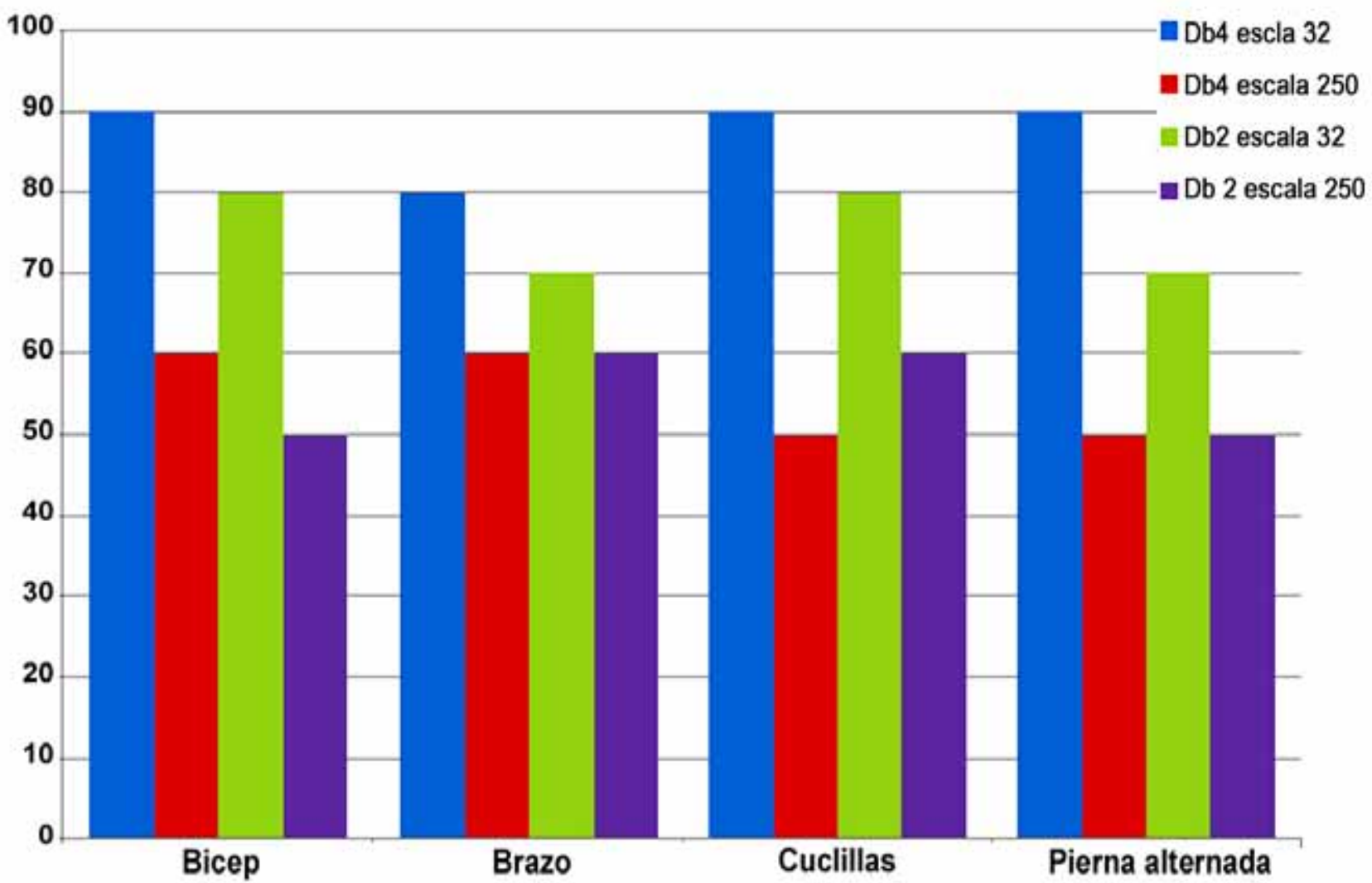

Figura 13. Efectividad (\%) de la predominancia entre CWT y real. 
mucha de esta información no es claramente visible, o no es notablemente visible. Por lo tanto, la representación en el dominio del tiempo no puede ser la mejor forma de analizar esta señal, en este caso, para este análisis se recomienda el uso de técnicas matemáticas más sofisticadas, la Transformada Wavelet es un ejemplo de estas técnicas.

Aunque la Transformada Wavelet tiene bajo costo computacional en el análisis, este costo puede ser muy alto si el número de escalas es muy alta. Por último, se llegó a la conclusión que la transformada wavelet puede ser aplicada en el procesamiento de señales EMGS.

\section{Conclusiones}

El prototipo del electromiógrafo de cuatro canales construido para este proyecto se puede observar en la Figura 14. Tiene un alcance máximo de treinta metros sin línea de vista entre el electromiógrafo y el computador con el módulo XBee receptor.

Se logró diseñar e implementar un electromiógrafo superficial de cuatro canales independientes en la adquisición, acondicionamiento y procesamiento DSP de las señales, utilizando diversos recursos como la recolección de la información, la prueba de diferentes componentes electrónicos, dando comodidad, fidelidad y precisión de las señales electromiográficas registradas.

Se utilizó el dispositivo XBee basado en el protocolo 802.15.4, con el fin de implementar la conectividad inalámbrica entre la etapa de digitalización de la señal y el PC con LabVIEW, esto con el fin de dar comodidad, libertad de movimiento del sujeto y aislamiento de alguna posible filtración de corriente que pueda ser perjudicial para la salud del sujeto conectado al electromiógrafo.

Se utilizó el software LabVIEW para visualizar los cuatro canales en el dominio del tiempo y procesarlas por CWT, el cual posee herramientas avanzadas para aplicaciones con complejidad matemática como lo es el procesamiento avanzado de señales, además tiene la facilidad de ser trabajado por el usuario, sin la necesidad de tener muchos conocimientos de programación al ser un lenguaje de programación gráfico.

Se realizaron las pruebas pertinentes para evaluar el desempeño del sistema bajo distintas condiciones, esto se logró probando el sistema en diferentes sujetos,

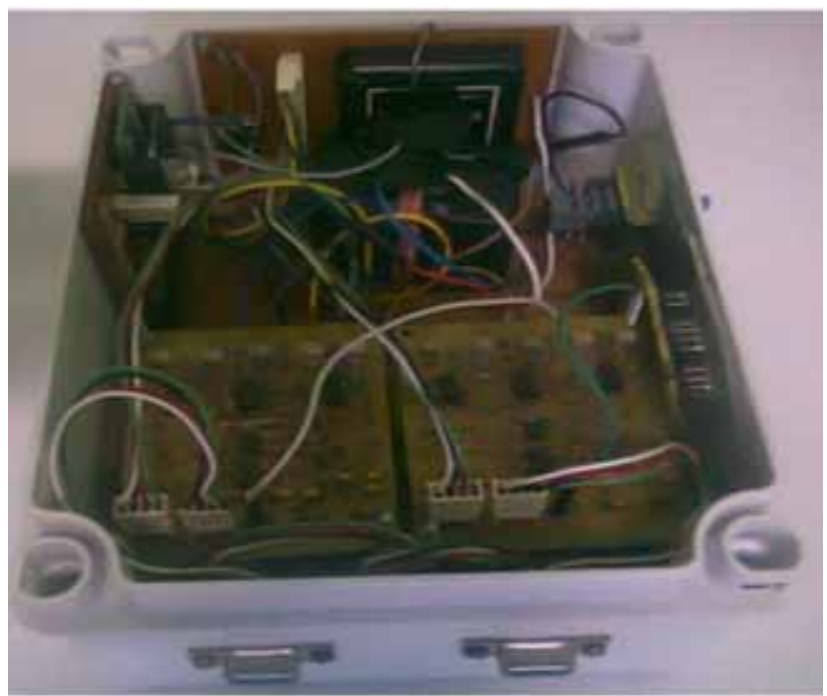

Figura 14. Prototipo electromiógrafo.

seleccionando las ganancias de los canales para las respectivas visualizaciones en LabVIEW, además se probó con distintas wavelet madres y escalas de la técnica matemática CWT permitiendo un análisis acertado.

Por medio de este proyecto se confirma la efectividad de la Transformada de Wavelet Continua (CWT) al superar las limitaciones y distorsiones de la Transformada de Fourier (TF), además soluciona el problema de resolución de la Transformada de Fourier Tiempo Corto (STFT), con el uso de una ventana variable en escala y traslación denominada wavelet madre, volviendo una señal no estacionara a una señal estacionaria, haciendo posible una mejor representación gráfica de la señal, con la capacidad de establecer componentes espectrales en una gráfica 3D denominada escalograma.

El electromiógrafo se puede usar en diferentes sujetos (personas en situación de discapacidad, accidentabilidad o atlética) para detectar las señales musculares en las extremidades superiores e inferiores, $y$ procesarlas para detectar diferentes enfermedades musculares, ya sea para diagnósticos médicos, desempeño deportivo o terapias físicas.

En trabajos futuros se recomienda realizar pruebas en sujetos con discapacidad neuromuscular y deportistas, en extremidades superiores e inferiores, aplicando distintas wavelets madres y escalas de la técnica matemática CWT, para análisis, detecciones de enfermedades musculares, lesiones y desempeño deportivo, con la asesoría de un neurólogo. 


\section{Referencias bibliograficas}

1. Carter, B., 2006. High-speed Notch filters. Consultado el 3 de junio 2014. http://www.ti.com/lit/an/ slyt235/slyt235.pdf

2. Chávez, C. M. A. Rodríguez, S. F. 2010. Revista Ingeniería Biomédica: Exoesqueletos para potenciar las capacidades humanas y apoyar la rehabilitación. Medellín, Colombia. Escuela de Ingeniería de Antioquia-Universidad CES. Medellín.

3. Fernández, J. Acevedo, R., 2007. Revista Ingeniería Biomédica: Influencia de la fatiga muscular en la señal electromiográfica de músculos estimulados eléctricamente, Escuela de Ingeniería de Antioquia, Medellín. ISSN 1794-1237

4. Muñoz, B. E. Paruma, O. Flórez, J. F., 2004. Aplicaciones de las señales mioeléctricas para el control de interfaces hombre-máquina, Universidad del cauca Popayán.
5. Pérez V. C. Zamanillo, J. M., 2007. Sistemas de telecomunicación. España. 91 pp.

6. Salazar, M., 2011. Observatorio Colombiano de Ciencia y Tecnología: Indicadores de ciencia y tecnología, Bogotá. ISBN 978-958-98956-6-5

7. Realpe, J. Jojoa, P. E. 2007., Análisis de señales emg superficiales y su aplicación en control de prótesis de mano. Volumen 4, núm. 1. Universidad del Cauca, Popayán.

8. Silva, C. W., 2005. Vibration and shock handbook. CRC press, USA. 11-4, 11-5. ISBN 0-8493-1580-8.

9. Soto, J. M., 2009. Ingeniería biomédica: Historia en construcción, Escuela de Ingeniería de Antioquia. Volumen 3, núm. 5. 1pp. Medellín.

10.TI., 2005. Ina128. Consultado el 10 de Junio de 2013. http://www.ti.com/lit/ds/symlink/ina128. Pdf. 11 pp. 
